Check for updates

Cite this: RSC Adv., 2019, 9, 9694

Received 7th January 2019

Accepted 21st March 2019

DOI: $10.1039 / \mathrm{c} 9 \mathrm{ra00124g}$

rsc.li/rsc-advances

\title{
A large-surface-area TS-1 nanocatalyst: a combination of nanoscale particle sizes and hierarchical micro/mesoporous structures $\uparrow$
}

\author{
Changlin Du, (D) ${ }^{\text {ab }}$ Nan Cui, ${ }^{\text {ab Linghao Li, }}$ ab Zile Hua (iD *ab and Jianlin Shi (D) ab
}

By a simple sequent process of dry-gel steam-assisted crystallization and following a top-down alkalietching treatment, hierarchically structured TS-1 nanozeolites (nanoTS-1_D) with abundant micro/ mesopores have been synthesized for the first time, and they exhibit remarkably high specific surface area of $606 \mathrm{~m}^{2} \mathrm{~g}^{-1}$ and pore volume of $0.86 \mathrm{~cm}^{3} \mathrm{~g}^{-1}$. Characterization by XRD, FTIR, UV-Vis and EM confirm the exclusive incorporation of titanium species in zeolite frameworks. More importantly, compared with the microporous TS-1 nanocrystal material with identical particle sizes of $80 \mathrm{~nm}$ (nanoTS-1) and submicrometer-sized mesoporous TS-1 material (mesoTS-1), here the reported nanoTS1_D catalyst shows greatly improved performance in the model reaction of 1-hexene epoxidation. $40.9 \%$ olefin conversion and $96.3 \%$ epoxide selectivity are achieved and its high stability is verified by the 6 recycling-regeneration tests.

\section{Introduction}

Owing to the high catalytic activity and stability towards many selective oxidation reactions using dilute $\mathrm{H}_{2} \mathrm{O}_{2}$ solution as the oxidant, the successful synthesis of MFI-type titanosilicate zeolites (TS-1) has been considered as a breakthrough in the field of microporous catalytic materials. ${ }^{\mathbf{1}, 2}$ However, restricted by the small pore size $(\sim 0.54 \mathrm{~nm}$ in pore width) of the microporous structures, conventional microporous TS- 1 materials show limited accessibility to the active titanium species located within the frameworks especially for bulky molecules, which is also a common issue to all types of microporous zeolites. ${ }^{3}$ In order to tackle these problems, two major strategies, i.e., decreasing zeolite particle sizes to the nanoscale (nanozeolites $)^{4,5}$ and introducing auxiliary mesoporous structures (hierarchical zeolites), ${ }^{6-8}$ have been developed and great progress has been made in recent years. It is believed that their superior performances are associated with the shortening of mass diffusion length in the microporous channels and the improved accessibility to the framework active sites. ${ }^{\mathbf{9}, 10}$ Unfortunately, synthesis of nanozeolites often suffers from the low solid yields and the consumption of a large amount of microporous structure-directing agents (SDAs). ${ }^{11}$ Meanwhile,

${ }^{a}$ State Key Lab of High Performance Ceramics and Superfine Microstructure, Shanghai Institute of Ceramics, Chinese Academy of Sciences, Shanghai 200050, China. E-mail: huazl@mail.sic.ac.cn

${ }^{b}$ Centre of Materials Science and Optoelectronics Engineering, University of Chinese Academy of Sciences, Beijing 100049, China

† Electronic supplementary information (ESI) available. See DOI: 10.1039/c9ra00124g additional mesopore templates, such as surfactants, ${ }^{\mathbf{1 2 , 1 3}}$ carbon materials $\mathbf{s}^{\mathbf{1 4 , 1 5}}$ and organosilanes ${ }^{\mathbf{1 6 , 1 7}}$ are necessarily adopted in the conventional synthesis of hierarchical zeolites. Based on the micro-/mesopore bi-functional diquaternary ammonium surfactant template design, Ryoo et al. reported a pioneering work on the preparation of single-unit-cell zeolite. ${ }^{18,19}$ The resultant TS-1 nanosheets showed high specific surface area of $580 \mathrm{~m}^{2} \mathrm{~g}^{-1}$ and notable catalytic activities with high epoxide selectivity for bulky olefin epoxidation. ${ }^{19}$ Very recently, Yu et al. prepared anatase-free nanosized hierarchical TS-1 zeolites by using Triton X-100 as mesoporous template under rotational crystallization conditions, which exhibits high catalytic performance in the alkene epoxidation reactions, e.g., 1-hexene. ${ }^{20}$ However, all these processes involve a large amount of commercial or home-made mesoporous template agents, which not only increase the cost of synthesis, but also result in the significant emission of greenhouse gas during the subsequent template elimination. On the other hand, as an alternative, post-synthesis demetallation through acid/alkali/steaming treatment or a combined process has been developed to be a general and facile approach for the preparation of aluminosilicate hierarchical zeolites. ${ }^{21-23}$ Different from negatively charged aluminosilicate frameworks, TS- 1 zeolites possess the neutral frameworks due to the equal valent substitution of Si by Ti. Thus, the post-synthesis alkali-etching treatment on TS-1 zeolites leads to nonselective removal between $\mathrm{Si}$ and $\mathrm{Ti}$ species from the framework in the same step, which implies that the variation of framework chemical compositions before and after alkali-etching treatment is controllable, as reported by Tuel et al. in preparing hollow TS-1 crystals through postsynthesis treatment with aqueous solutions of 
tetrapropylammonium hydroxide. ${ }^{23}$ Moreover, it is suggested that the dissolution/recrystallization process helps the reincorporation of extra-framework $\mathrm{Ti}$ species into the zeolite frameworks, which contributes to the improvement of materials catalytic activity. In the report of Gläser et al., a two-step desilication and surfactant-assisted recrystallization approach was adopted for preparing nanosized TS-1 with micro-/mesoporosity. ${ }^{24}$ The resultant material shows specific surface area of $503 \mathrm{~m}^{2} \mathrm{~g}^{-1}$ and enhanced activity in the epoxidation of the unsaturated fatty acid methyl esters (FAME) with $\mathrm{H}_{2} \mathrm{O}_{2}$. Though highly facile and effective, there are still extremely few reports of preparation of hierarchical TS-1 zeolites using such a postetching treatment, which is expected here to be applicable for the synthesis of hierarchically structured TS-1 nanozeolites with ever-larger specific surface area and much enhanced catalytic activity.

In our previous report, TS-1 nanozeolites (nanoTS-1) with uniform and adjustable particle sizes have been successfully prepared via a dry-gel steam-assisted crystallization (SAC) procedure. $^{25}$ Herein, by a combination with post-synthesis alkali-etching treatment, hierarchically structured TS-1 nanozeolites (nanoTS-1_D) with abundant micro-/mesopores have been synthesized for the first time, which exhibit an extremely high specific surface area of $606 \mathrm{~m}^{2} \mathrm{~g}^{-1}$ and total pore volume of $0.86 \mathrm{~cm}^{3} \mathrm{~g}^{-1}$, even higher than those of reported TS-1 nanosheets. ${ }^{19}$ Importantly, this unique material exhibits elevated catalytic activity in 1-hexene epoxidation, which is superior to the conventional micrometer-sized TS-1 (microTS-1), nanoTS-1 precursors with identical particle sizes of $80 \mathrm{~nm}$, and submicrometer-sized mesoporous TS-1 materials (mesoTS-1) synthesized by direct hydrothermal process with the assistance of cetyltrimethyl-ammonium bromide (CTAB) surfactant and ethanol. ${ }^{13}$

\section{Experimental}

\subsection{Catalysts preparation}

2.1.1 Nano-crystalline TS-1 zeolite. Nano-crystalline TS-1 zeolites were synthesized according to the steam-assisted crystallization process with a permanent $\mathrm{Si} / \mathrm{Ti}$ molar ratio of 50 by controlling the molar ratio of tetraethyl orthosilicate (TEOS) and tetrabutyl titanate (ТВОT) in the preparation process. ${ }^{25}$ In the typical synthesis, $10.98 \mathrm{~g}$ of tetrapropylammonium hydroxide (TPAOH, $25 \mathrm{wt} \%$ ) was added dropwise into $10.42 \mathrm{~g}$ of TEOS together with $18 \mathrm{~g}$ of deionized water to obtain an aqueous solution. The mixture was stirred at $40{ }^{\circ} \mathrm{C}$ for $4 \mathrm{~h}$. Then, $0.34 \mathrm{~g}$ of TBOT dissolved in $6 \mathrm{~g}$ of isopropanol was added dropwise to the mixture and stirred below $4{ }^{\circ} \mathrm{C}$ for another $4 \mathrm{~h}$, which makes $\mathrm{Ti}$ incorporated into silicon tetrahedron. The mixture was stirred at $40{ }^{\circ} \mathrm{C}$ for $48 \mathrm{~h}$ to get the solid precursor gel, then crystallized at $180{ }^{\circ} \mathrm{C}$ for $10 \mathrm{~h}$. After repeated centrifugation, rinsing with deionized water and drying at $70{ }^{\circ} \mathrm{C}$ overnight, the precursor was calcined in air at $550{ }^{\circ} \mathrm{C}$ for $6 \mathrm{~h}$ to get nano-crystalline TS-1 zeolite (denoted as nanoTS-1).

2.1.2 Hierarchically structured nano-crystalline TS-1 zeolite. The alkali-etching procedure of nanoTS-1 was according to the methodology reported by Groen et al. ${ }^{30}$ The as- synthesized nanoTS-1 sample was treated with alkaline solution of $\mathrm{NaOH}$ and TPAOH. In the typical preparation, $2.5 \mathrm{~g}$ of nanoTS-1 was added into $75 \mathrm{ml}$ of alkaline solution $(0.08 \mathrm{M}$ $\mathrm{NaOH} / 0.12 \mathrm{M}$ TPAOH). Then, the mixture was stirred at $65{ }^{\circ} \mathrm{C}$ for $30 \mathrm{~min}$. After rapid cooling, repeated centrifugation, rinsing with deionized water to neutral, the sample was dried at $80{ }^{\circ} \mathrm{C}$ for $12 \mathrm{~h}$. The obtained sample was mixed under stirring with $250 \mathrm{ml}$ of aqueous $\mathrm{HCl}$ solution $(0.1 \mathrm{M})$. Then, the mixture was stirred at $65{ }^{\circ} \mathrm{C}$ for $6 \mathrm{~h}$. After rapid cooling, repeated centrifugation, rinsing with deionized water to neutral, the sample was dried at $80{ }^{\circ} \mathrm{C}$ for another $12 \mathrm{~h}$. Finally, after calcined in air at $540{ }^{\circ} \mathrm{C}$ for $5 \mathrm{~h}$, hierarchically structured nano-crystalline TS- 1 zeolite was obtained (denoted as nanoTS-1_D).

\subsection{Catalyst characterization techniques}

The XRD patterns were obtained on Rigaku D/Max 2200PC powder X-ray diffractometer with $\mathrm{Cu}_{\mathrm{K} \alpha}$ radiation source $(\lambda=$ $0.15418 \mathrm{~nm}$ ). The X-ray tube was operated at $40 \mathrm{kV}, 40 \mathrm{~mA}$; the two-theta angle was scanned from $5^{\circ}$ to $50^{\circ}$ (angular step wise $0.02^{\circ}$ ) at a speed of $5^{\circ} \min ^{-1}$. The surface area and pore size distribution of the catalysts were characterized on an automatic $\mathrm{N}_{2}$ adsorption-desorption instrument (TriStar II 3020) at $77 \mathrm{~K}$. The specific surface areas of the samples were calculated by the Brunauer-Emmett-Teller (BET) method and pore size distribution by the Barrett-Joyner-Halenda (BJH) model in the sorption branches of the isotherms. Before the measurement, the samples were dried at $150{ }^{\circ} \mathrm{C}$ for $5 \mathrm{~h}$. Diffuse reflectance UVvis spectra of the samples were recorded from 190 to $800 \mathrm{~nm}$ using a Shimadzu UV-310PC spectrometer with $\mathrm{BaSO}_{4}$ as a reference. And silicon, titanium contents of the catalysts were measured by optical emission spectrometry with inductively coupled plasma (ICP-OES) on Agilent 725. Field-emission scanning electron microscopy (FE-SEM) measurements of the samples were characterized on HITACHI SU8220. Besides, Transmission Electron Microscopy (TEM) pictures were carried out on JEM-2100F electron microscope equipped with a field emission source operating at $200 \mathrm{kV}$. The FT-IR spectra were obtained on a Fourier Transform Infrared Spectroscopy (FT-IR) measurement FTIR-7600 to analyze the existence form and coordination status of titanium.

\subsection{Catalytic evaluation}

The epoxidation of 1-hexene with $\mathrm{H}_{2} \mathrm{O}_{2}$ (30 wt\% aqueous solution) was carried out in a three-necked round-bottomed flask equipped with a reflux condenser. Typically, $50 \mathrm{mg}$ of catalyst, $10 \mathrm{ml}$ of methanol, $10 \mathrm{mmol}$ of 1 -hexene and $10 \mathrm{mmol}$ of $\mathrm{H}_{2} \mathrm{O}_{2}$ were mixed in the reactor and stirred vigorously at $60{ }^{\circ} \mathrm{C}$ for $4 \mathrm{~h}$. After reaction, the products were characterized on Shimadzu GC/ MS-2010 equipped with a Rxi-5Sil MS column and an FID detector. To characterize the catalyst stability, we separated the used nanoTS-1_D by centrifugation and rinsing with deionized water and ethanol after each reaction. Then the recovered catalyst was dried at $70{ }^{\circ} \mathrm{C}$ overnight, following by calcined in air at $540{ }^{\circ} \mathrm{C}$ for $3 \mathrm{~h}$. Finally, the regenerated nanoTS-1_D (denoted as Re. nanoTS-1_D) was obtained and kept for next recycling catalytic reaction. 6 recycling regeneration tests have been performed. 


\section{Results and discussion}

\subsection{Catalysts characterization}

3.1.1 XRD analysis. The powder X-ray diffraction (XRD) patterns of synthesized TS-1 zeolites are presented in Fig. 1A. All of them reveal the typical Bragg diffraction peaks of MFI-type zeolites. Meanwhile, the absence of diffraction peak at $2 \theta$ of $25.40^{\circ}$, which is associated with the (101) plane of $\mathrm{TiO}_{2}$ anatase phases, confirms that XRD-detectable extra-framework titanium is absent in the resultant materials and titanium is incorporated into the siliceous zeolite framework. More evidences could be found in the following spectroscopic analyses (UV-vis spectra of Fig. 1B of and FT-IR spectra of Fig. S3†). Except for sample nanoTS-1_D, the comparable high-intensity diffraction peaks with the other three samples prove their high crystallinity. About nanoTS-1_D, a reduction in the diffraction intensity could be resulted from the auxiliary intracrystal mesoporous structures which improve the mass transfer in the hierarchical zeolites, but as an artificial defect break the intrinsic periodic structure of microporous zeolite crystals. ${ }^{24}$ In the meantime, the coordination status of Ti species was also characterized by UV-vis spectrometer, as shown in Fig. 1B. All TS-1 materials present an obvious absorbance at $220 \mathrm{~nm}$ ascribed to the charge transition from $\mathrm{O}(2 \mathrm{p})$ to $\mathrm{Ti}(3 \mathrm{~d}),{ }^{26}$ which clearly demonstrates that most of Ti species have been tetrahedrally coordinated in the siliceous zeolite framework. It should be noted that a weak absorption edge onset appears at $\sim 320 \mathrm{~nm}$ (the inset dashed rectangle) in samples nanoTS-1 and
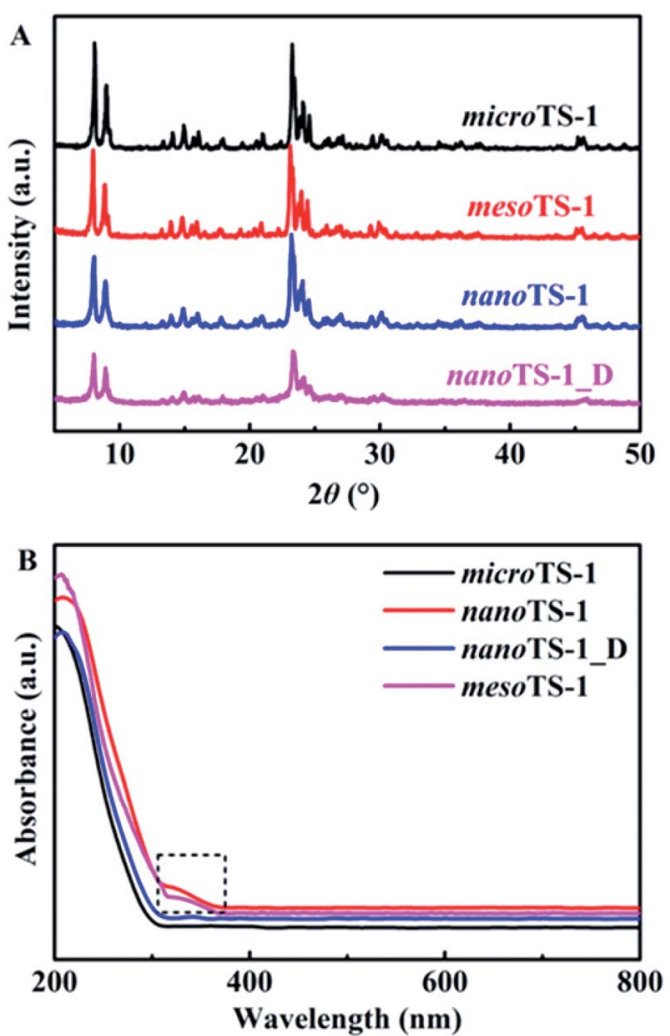

Fig. 1 (A) Powder XRD patterns and (B) UV-vis spectra of synthesized TS-1 zeolites.
mesoTS-1, which is associated with titanium-enriched species or titania nanoclusters, verifying the presence of extraframework Ti species in the samples, though it is undetectable with XRD characterization. In contrast, such a weak absorption edge is not visible for sample nanoTS-1_D, which means that the extra-framework titanium species has been removed through the post-synthesis alkali-etching process. As a result, a slightly increased $\mathrm{Si} / \mathrm{Ti}$ molar ratio from 72 to 82 is obtained for sample nanoTS-1_D, as listed in Table 1. The clear adsorption band at $960 \mathrm{~cm}^{-1}$ in Fig. S3† of FT-IR spectra, which is assigned to the stretching vibration of $\mathrm{Ti}-\mathrm{O}-\mathrm{Si}$ bond, provides an additional proof for the successful incorporation of Ti into synthesized zeolite frameworks in the samples.,27,28

3.1.2 Nitrogen sorption/desorption analysis. The $\mathrm{N}_{2}$ sorption isotherms and $\mathrm{BJH}$ pore size distributions (PSD) of the synthesized TS-1 zeolites are shown in Fig. 2, and their textural properties are summarized in Table 1 . As expected, microTS-1 zeolite shows the typical type I profile of microporous materials with a high uptake at low relative pressures $\left(P / P_{0}<0.1\right)$ and a plateau at high relative pressures. Consequently, its PSD plot based on the adsorption branch demonstrates the peak absence in the mesopore range of $2.0-100 \mathrm{~nm}$. The corresponding specific surface area and total pore volume are $420 \mathrm{~m}^{2} \mathrm{~g}^{-1}$ and $0.22 \mathrm{~cm}^{3} \mathrm{~g}^{-1}$, respectively. For sample nanoTS-1 and mesoTS-1, the type IV isotherms are present and the apparent uptake steps at $P / P_{0}$ of 0.90 and 0.40 respectively reflects their specific intercrystal and intracrystal mesoporous structures, which are in agreement with previous reports. ${ }^{\mathbf{1 3 , 2 5}}$ Interesting results are found for alkali-etched sample nanoTS-1_D. Compared to that of nanoTS-1 precursors, besides the hysteresis loop and steep rise at the high relative pressures of 0.80-0.99 associated with the intercrystal mesopores, the $\mathrm{N}_{2}$ sorption volume is significantly increased in the whole pressure range and a new hysteresis loop appears at the relative pressures between 0.450.70 , indicating the formation of new mesoporous structures. A double-peak distribution is observed in its PSD plot, in which the mesopores centred at ca. $44.3 \mathrm{~nm}$ are the intercrystal mesovoids and the newly appeared small peak centred at $c a .3 .8 \mathrm{~nm}$ corresponds to the alkali-etching generated intracrystal mesopores. As listed in Table 1, sample nanoTS-1_D possesses the highest specific surface area of $606 \mathrm{~m}^{2} \mathrm{~g}^{-1}$ and total pore volume of $0.86 \mathrm{~cm}^{3} \mathrm{~g}^{-1}$, which are respectively $122 \mathrm{~m}^{2} \mathrm{~g}^{-1}$ and $0.44 \mathrm{~cm}^{3} \mathrm{~g}^{-1}$ higher than those of nanoTs- 1 precursor, and also higher than the corresponding values (specific surface area of $580 \mathrm{~m}^{2} \mathrm{~g}^{-1}$ and total pore volume of $0.61 \mathrm{~cm}^{3} \mathrm{~g}^{-1}$ ) of single-unitcell TS-1 nanosheets. ${ }^{19}$

3.1.3 EM analysis. TEM and SEM images provide the direct evidences for the formation of intracrystal mesopores in nanoTS-1_D materials. SEM images of Fig. 3C and S1B $\dagger$ depict that sample nanoTS-1_D consists of highly dispersed and uniform nanocrystals of $c a .80 \mathrm{~nm}$, similar to that of nanoTS-1 precursor as shown in Fig. 3A and S1A. $\dagger$ Additionally, the inset histograms of DLS analyses in Fig. S1A and $\mathrm{B} \uparrow$ show the similar particle size distributions and the mean particle sizes are $c a$. $80 \mathrm{~nm}$. The consistency between SEM and DLS results confirms the negligible effect of alkali-etching treatment on the particle size of TS-1 nanozeolites. On the other hand, there exists 
Table 1 Chemical composition and structural characteristics of the synthesized TS-1 zeolites

\begin{tabular}{llllll}
\hline Sample & $\mathrm{Si} \mathrm{Ti}^{a}$ & $S_{\text {BET }}\left(\mathrm{m}^{2} \mathrm{~g}^{-1}\right)$ & $S_{\text {exter }}\left(\mathrm{m}^{2} \mathrm{~g}^{-1}\right)$ & $V_{\text {total }}\left(\mathrm{cm}^{3} \mathrm{~g}^{-1}\right)$ & 0.22 \\
microTS-1 & 75 & 420 & 136 & 0.36 & 0.10 \\
mesoTS-1 & 64 & 511 & 351 & 0.42 & 0.29 \\
nanoTS-1 & 72 & 484 & 202 & 0.86 & 0.30
\end{tabular}

${ }^{a} \mathrm{Si} / \mathrm{Ti}$ molar ratio in the bulk determined by ICP.
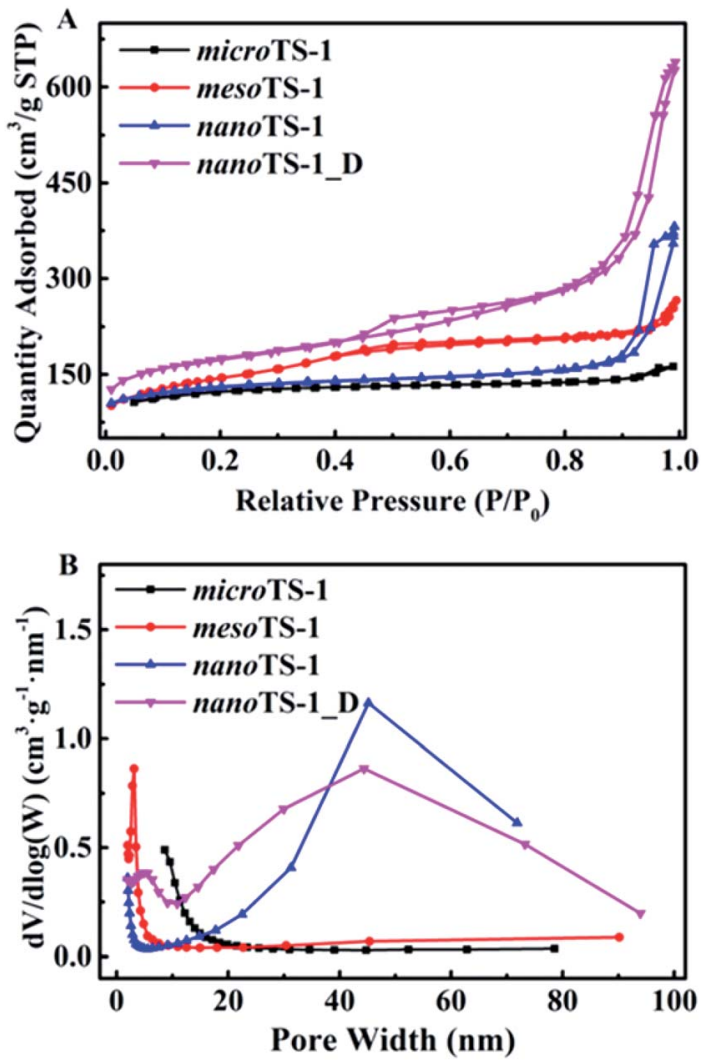

Fig. 2 (A) $\mathrm{N}_{2}$ sorption isotherms and (B) PSD of synthesized TS-1 zeolites.

significant difference on the particle morphology between synthesized nanoTS-1 and nanoTS-1_D, i.e., compared to the smooth surface of nanoTS-1, the particle surface of nanoTS-1_D is rough with abundant mesopores of about $2.8-4.6 \mathrm{~nm}$ on the surface. Moreover, as shown in the TEM images of Fig. 3D, compared to the homogeneous structure of sample nanoTS- 1 of Fig. 3B, nanoTS-1_D exhibits much more significant and abundant pore-structures, which is believed to result from the additional mesoporous structures induced by the following alkali-etching treatment. The mesopore sizes are $c a .3 .5 \mathrm{~nm}$, which are consistent with above $\mathrm{N}_{2}$ sorption and SEM results.

\subsection{Catalytic performance}

In the model reaction of 1-hexene epoxidation, all synthesized TS1 zeolites show the approximately similar product distributions and the target 1,2-epoxyhexane selectivities are larger than 95\%, as listed in Table 2. However, their catalytic activities are substantially distinguishable from each other. Since all synthesized TS-1 materials possess the similar Si/Ti ratio (as listed in Table 1), the great difference on catalyst activities should be related to their specific textural properties. Among them, microTS-1 exhibits the lowest 1-hexene conversion of $19.7 \%$, which is in accordance with reported results and confirms the limited accessibility of the reactants to the framework titanium active centres in the conventional microporous catalysts. ${ }^{29}$ As expected, the 1-hexene conversions on samples nanoTS-1 and mesoTS- 1 are slightly higher at $23.0 \%$ and $24.5 \%$ respectively because of their auxiliary intercrystal or intracrystal mesoporous structures. Interestingly, benefitting from the extraordinarily high surface area of alkali-etched nanoTS-1, a significantly elevated conversion of $40.9 \%$ has been achieved, which is more than twice that of microTS-1. And the calculated TON also certifies the good accessibility of surface titanium active centres in synthesized nanoTS-1_D and consequent high activity. Meanwhile, it should be noted that the extra-framework titanium species would cause the non-catalytic decomposition of $\mathrm{H}_{2} \mathrm{O}_{2}$ oxidant and are harmful to the catalytic oxidation reactions. ${ }^{20}$ As suggested by the above UV-vis results, the elimination of extra-framework Ti species also contributes to the activity enhancement of sample nanoTS-1_D.

In addition, recycling experiments were performed to verify the catalytic stability of synthesized nanoTS-1_D. As shown in Table 2 and S1, $\uparrow$ the 1-hexene conversion and 1,2-epoxyhexane selectivity keep almost constant in the consecutive 6 recyclingregeneration tests, demonstrating the high stability of nanoTS1_D catalysts. Moreover, XRD patterns also indicates the well-

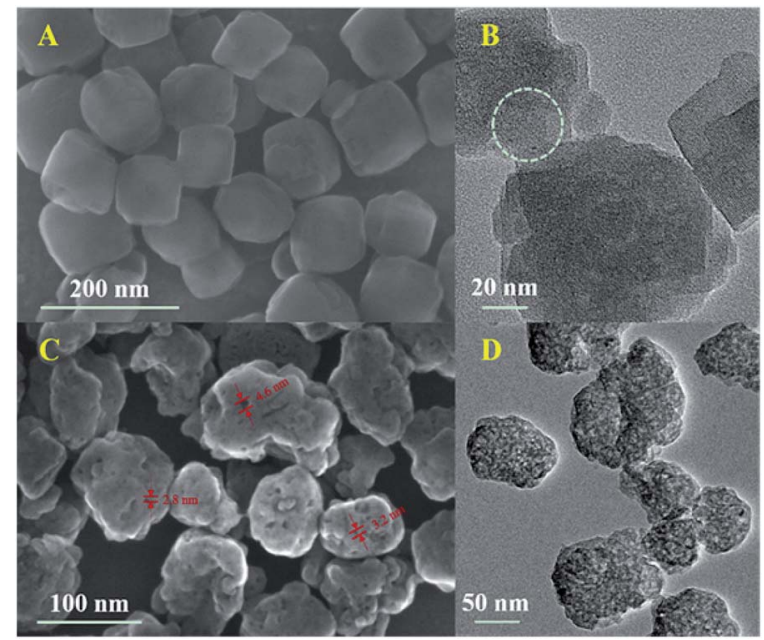

Fig. 3 (A) SEM image and (B) TEM image of synthesized nanoTS-1; (C) SEM image and (D) TEM image of synthesized nanoTS-1_D. 
Table 2 Catalytic performances of synthesized TS-1 zeolites in the epoxidation reaction of 1-hexene using $\mathrm{H}_{2} \mathrm{O}_{2}{ }^{a}$

\begin{tabular}{lllll}
\hline & & \multicolumn{3}{l}{ 1-Hexene epoxidation } \\
\cline { 3 - 5 } Sample & Ti content $^{b}(\mathrm{wt} \%)$ & Conv. (\%) & Sel. $^{c}(\%)$ & TON \\
\hline microTS-1 $_{\text {nanoTS-1 }}$ & 1.05 & 19.7 & 96.4 & 180 \\
mesoTS-1 $_{\text {nanoTS-1_D }}$ & 1.09 & 23.0 & 95.5 & 202 \\
Re. nanoTS-1_D $^{d}$ & 0.96 & 24.5 & 96.5 & 191 \\
nanyyyy & 0.94 & 40.9 & 96.3 & 408 \\
& & 40.7 & 95.9 & 415
\end{tabular}

${ }^{a}$ Reaction conditions: 1-hexene, $10 \mathrm{mmol} ; \mathrm{H}_{2} \mathrm{O}_{2}, 10 \mathrm{mmol} ; \mathrm{MeOH}$, $10 \mathrm{ml}$; catalyst, $50 \mathrm{mg} ; 60{ }^{\circ} \mathrm{C}, 4 \mathrm{~h} .{ }^{b} \mathrm{Ti}$ content in the bulk catalysts determined by ICP. ${ }^{c}$ The selectivity of 1,2-epoxyhexane. ${ }^{d}$ nanoTS-1_D catalyst after 6 recycling-regeneration tests in the epoxidation of 1hexene under the same reaction condition.

preserved MFI-type structure of nanoTS-1_D after 6 recyclingregeneration (Fig. $\mathrm{S} 3 \mathrm{~A} \dagger$ ). Meanwhile, UV-vis spectra suggest that no anatase $\mathrm{TiO}_{2}$ is present in the regenerated nanoTS-1_D material (Fig. $\mathrm{S} 3 \mathrm{~B} \dagger$ ). Besides, the $\mathrm{N}_{2}$ sorption isotherms and BJH PSD results of the regenerated nanoTS-1_D are consistent with those of the original material, indicating the wellmaintained hierarchically micro-/mesoporous structure in recycled nanoTS-1_D (Fig. S3C, D and Table S1†). All of these characterization results demonstrate that the large-surface-area nanoTS-1_D zeolite with nanoscale particle size and hierarchically micro-/mesoporous structure is an extremely active and stable catalyst in epoxidation of 1-hexene.

\section{Conclusion}

In summary, a novel nano-sized and hierarchical micro-/ mesoporous structured TS-1 nanocatalyst (nanoTS-1_D) has been prepared by a facile sequent process of dry-gel steamassisted crystallization and following alkali-etching treatment. The hierarchical nanocatalyst possesses remarkably high specific surface area of $606 \mathrm{~m}^{2} \mathrm{~g}^{-1}$ and total pore volume of 0.86 $\mathrm{cm}^{3} \mathrm{~g}^{-1}$, which are comparable to the previously reported single-unit-cell TS-1 nanosheets. As a result, in the model reaction of 1-hexene epoxidation, nanocatalyst demonstrates $40.9 \%$ of olefin conversion and $96.3 \%$ of epoxide selectivity, which is superior to the conventional micrometer-sized TS-1 (microTS-1), nanoTS-1 precursors with identical particle sizes of $80 \mathrm{~nm}$, and submicrometer-sized mesoporous TS- 1 materials of mesoTS-1. Considering the wide applications of titaniumsilicate zeolites in the selective oxidation reactions, such a facile but efficient two-step strategy for the synthesis of novelstructured TS-1-based catalysts would be of high significance in the fine chemical production and petrochemical industry. Further studies about detailed processes and mechanisms of post-synthesis alkali-etching and its effect on the textural and surface properties of TS-1 and non-aluminum doping silicate zeolites are in progress.

\section{Conflicts of interest}

There are no conflicts to declare.

\section{Acknowledgements}

This work was sponsored by the National Natural Science Foundation of China (21776297, U1510107).

\section{Notes and references}

1 U. Wilkenhöner, G. Langhendries, F. V. Laar, G. V. Baron, D. W. Gammon, P. A. Jacobs and E. V. Steen, J. Catal., 2001, 203, 201.

2 D. P. Serrano, R. Sanz, P. Pizarro, I. Moreno and S. Medina, Appl. Catal., B, 2014, 146, 35.

3 X. Zhang, D. Liu, D. Xu, S. Asahina, K. A. Cychosz and K. Varoon, Science, 2012, 336, 1684.

4 W. Schwieger, A. G. Machoke, T. Weissenberger, A. Inayat, T. Selvam, M. Klumpp and A. Inayat, Chem. Soc. Rev., 2016, 45, 3353.

5 P. Bai, E. Haldoupis, P. J. Dauenhauer, M. Tsapatsis and J. I. Siepmann, ACS Nano, 2016, 10, 7612.

6 D. P. Serrano, J. M. Escola and P. Pizarro, Chem. Soc. Rev., 2013, 42, 4004.

7 B. Li, Z. Hu, B. Kong, J. Wang, W. Li, X. Sun, X. Qian, Y. Yang, W. Shen, H. Xu and D. Zhao, Chem. Sci., 2014, 5, 1565.

8 M. Milina, S. Mitchell, P. Crivelli, D. Cooke and J. PérezRamírez, Nat. Commun., 2014, 5, 3922.

9 D. Verboekend and J. Pérez-Ramírez, Catal. Sci. Technol., 2011, 1, 879.

10 H. Zhang, Z. Hu, L. Huang, H. Zhang, K. Song, L. Wang, Z. Shi, J. Ma, Y. Zhuang, W. Shen, Y. Zhang, H. Xu and Y. Tang, ACS Catal., 2015, 5, 2548.

11 R. Martinez-Franco, C. Paris, M. E. Cristina, C. Martinez, M. Moliner and A. Corma, Chem. Sci., 2015, 7, 102.

12 K. Na, C. Jo, J. Kim, K. Cho, J. Jung, Y. Seo, R. J. Messinger, B. F. Chmelka and R. Ryoo, Science, 2011, 333, 328.

13 Y. Zhu, Z. Hua, X. Zhou, Y. Song, Y. Gong, J. Zhou, J. Zhao and J. Shi, RSC Adv., 2013, 3, 4193.

14 W. Ying, E. Tanja, P. Krijin and J. Zečević, Chem. Soc. Rev., 2015, 44, 7234.

15 H. Chen, J. Wydra, X. Zhang, P. Lee, Z. Wang, W. Fan and M. Tsapatsis, J. Am. Chem. Soc., 2013, 133, 12390.

16 Y. Zhang, K. Zhu, X. Duan, P. Li, X. Zhou and W. Yuan, RSC Adv., 2014, 4, 14471.

17 P. Wu, M. Yang, W. Zhang, S. Xu, P. Tian and Z. Liu, Chem. Commun., 2017, 53, 4985.

18 M. Choi, K. Na, J. Kim, Y. Sakamoto, O. Terasaki and R. Ryoo, Nature, 2009, 461, 246.

19 K. Na, C. Jo, J. Kim, W. Ahn and R. Ryoo, ACS Catal., 2011, 1, 901.

20 T. Zhang, X. Chen, G. Chen, M. Chen, R. Bai, M. Jia and J. Yu, J. Mater. Chem. A, 2018, 6, 9473.

21 D. Wang, L. Zhang, L. Chen, H. Wu and P. Wu, J. Mater. Chem. A, 2015, 3, 3511.

22 K. A. Tarach, J. Martinez-Triguero, F. Rey and K. Góra-Marek, J. Catal., 2016, 339, 256.

23 Y. Wang, M. Lin and A. Tuel, Microporous Mesoporous Mater., 2007, 102, 80. 
24 N. Wilde, M. Pelz, S. Gebhardt and R. Gläser, Green Chem., 2015, 17, 3378.

25 T. Ge, Z. Hua, J. Lv, J. Zhou, H. Guo, J. Zhou and J. Shi, CrystEngComm, 2017, 19, 1370.

26 Y. Cheneviere, F. Chieux, V. Caps and A. Tuel, J. Catal., 2010, 269, 161.

27 A. Kumar, D. Srinivas, P. Pizarro, I. Moreno and S. Medina, J. Catal., 2012, 293, 126.
28 J. Li, J. Li, Z. Zhao, X. Fan, J. Liu, Y. Wei, A. Duan, Z. Xie and Q. Liu, J. Catal., 2017, 352, 361.

29 L. Wang, J. Sun, X. Meng, W. Zhang, J. Zhang, S. Pan, Z. Shen and F. Xiao, Chem. Commun., 2014, 50, 2012.

30 J. Groen, L. Peffer, J. Moulijn and J. Pérez-Ramírez, Microporous Mesoporous Mater., 2004, 69, 29. 